\title{
A new precoded partial transit sequence method using the discrete cosine transform for peak-to-average power ratio reduction in an orthogonal frequency division multiplexing system
}

\author{
Adnan Haider Yusef Sa'd*, Aeizaal Azman Abd. Wahab, Hisham Haider Yusef Sa'ad \\ School of Electrical and Electronic Engineering, Universiti Sains Malaysia, 14300 Nibong Tebal, \\ Pulau Pinang, Malaysia
}

*Corresponding author, e-mail: eng.adnanhaider@gmail.com

Received 25 Oct 2015

Accepted 31 Dec 2016

\begin{abstract}
Orthogonal frequency division multiplexing (OFDM) is a well-known transmission technique that is adopted in a wide range of standards these days mainly because of its high data bit rate and spectral efficiency. However, OFDM has one major problem called the peak-to-average power ratio (PAPR). This paper will introduce a new method that uses the discrete cosine transform to enhance the PAPR reduction over that achieved in the partial transmit sequence (PTS) approach. The maximum achieved improvement of PAPR reduction compared to the conventional PTS approach is $257 \%$ with only 4 iterations and the maximum PAPR reduction achieved in the simulation is $5.7 \mathrm{~dB}$.
\end{abstract}

KEYWORDS: PTS, OFDM, PAPR

\section{INTRODUCTION}

Orthogonal frequency-division multiplexing (OFDM) is a digital encoding method which uses multiple narrowband subcarriers instead of a single wide-band carrier. This means that more data can be transmitted. The orthogonality feature of OFDM allows adjacent channels to overlap which results in maximizing the data bit rate and spectral efficiency. In addition, OFDM is able to combat multipath fading and inter-symbol interference issues using a simple equalizer. As a result, OFDM is adopted in lots of wired and wireless communication standards such as DVB, DSL, 802.11g, 802.11a, and LTE.

At first sight, OFDM appears as the best technique for transmission. However, it suffers from one major problem called the high peak-to-average power ratio (PAPR). OFDM creates an output signal with very high peaks. The high peaks in the OFDM signal mostly cannot be amplified linearly using a normal power amplifier. Hence a complex power amplifier design with backoffs is needed to maintain linearity in order to keep the OFDM signal undistorted.

There are many techniques proposed for reducing PAPR. Some degrade communication performance such as clipping and some use distortionless methods to reduce PAPR. Distortionless methods such as partial transmit sequence (PTS) ${ }^{1-3}$, selected mapping $(\mathrm{SLM})^{1}$, interleaving $^{4}$, tone injection ${ }^{5,6}$, tone reservation $^{6-9}$, and the spread technique ${ }^{10}$ are the feasible options for reliable communication.

In this paper, a new pre-coding technique is proposed along with PTS to enhance the performance of the PTS technique. The discrete cosine transform (DCT) is known for its energy compaction and it has been used in many applications such as compressing images in the JPEG standard ${ }^{11}$.

It is not the first time that DCT has been used to reduce PAPR ${ }^{12-15}$. Here the inverse DCT (IDCT) is used as a precoder for PAPR reduction where each value of the partial input data sequence is spread over all subcarriers using the IDCT. In other words, each partial data sequence has its time-domain signal values of DCT spread over all subcarriers using the IDCT.

\section{PEAK-TO-AVERAGE POWER RATIO (PAPR)}

If $X=\left[X_{0}, X_{1}, \ldots, X_{N-1}\right]^{\mathrm{T}}$ is the complex sequence which represents the OFDM input data in the frequency domain, then the OFDM signal in the time domain $x(t)$ can be represented using the inverse discrete Fourier transform (IDFT):

$$
x(t)=\frac{1}{\sqrt{N}} \sum_{k=0}^{N-1} X_{k} \mathrm{e}^{\mathrm{i} 2 \pi k \Delta f t}, 0 \leqslant t<T_{\mathrm{s}}
$$




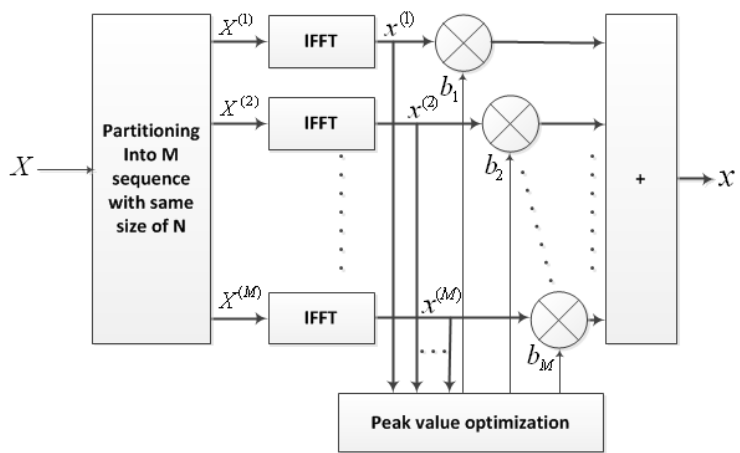

Fig. 1 PTS block diagram.

where $N$ is the total number of subcarriers, $X_{k}$ is the input data symbol of the subcarrier $k, \Delta f$ is the frequency difference between adjacent subcarriers, and $T_{s}=1 / \Delta f$ is the symbol duration. In implementation, the inverse fast Fourier transform (IFFT) algorithm is mostly used in place of the IDFT.

The PAPR of the OFDM signal is defined as the ratio of OFDM signal power peak value to its average power value ${ }^{16}$ and is found from

$$
\operatorname{PAPR}=\frac{\max \left|x_{k}\right|^{2}}{E\left[\left|x_{k}\right|^{2}\right]}, \quad k=0,1, \ldots, N L-1
$$

where $E$ denotes expectation operator and $L$ is the oversampling value. PAPR for any proposed system can be evaluated using the complementary cumulative distribution function (CCDF) which is given by

$$
\mathrm{CCDF}=\operatorname{Pr}\left(\mathrm{PAPR}>\mathrm{PAPR}_{0}\right)
$$

where Pr denotes probability.

\section{PARTIAL TRANSMIT SEQUENCE (PTS)}

In this method, the complex input sequence $X=$ $\left[X_{0}, X_{1}, \ldots, X_{N-1}\right]^{\mathrm{T}}$ is partitioned into $M$ disjoint partitions where each partition $X^{(m)}$ is applied to a length- $N$ IFFT subblock. The partial output $x^{(m)}$ is multiplied by a phase rotation factor $b_{\mathrm{m}}$ where $b_{\mathrm{m}}=\mathrm{e}^{j \phi_{\mathrm{m}}}$ and $\phi_{\mathrm{m}} \in[0,2 \pi]$. Fig. 1 shows the block diagram for the PTS approach.

In PTS, the number of admitted angles $W$ is reduced to reduce the capacity loss. Hence it is recommended that $W$ is not more than 4 . Also, to simplify the implementation, it is recommended to have $b_{\mathrm{m}} \in\{ \pm 1\}$ or $b_{\mathrm{m}} \in\{ \pm 1, \pm j\}$. The value of $b_{\mathrm{m}}$ should be chosen carefully so that the summation result of all rotated partial outputs has an acceptably low PAPR. The transmitter should

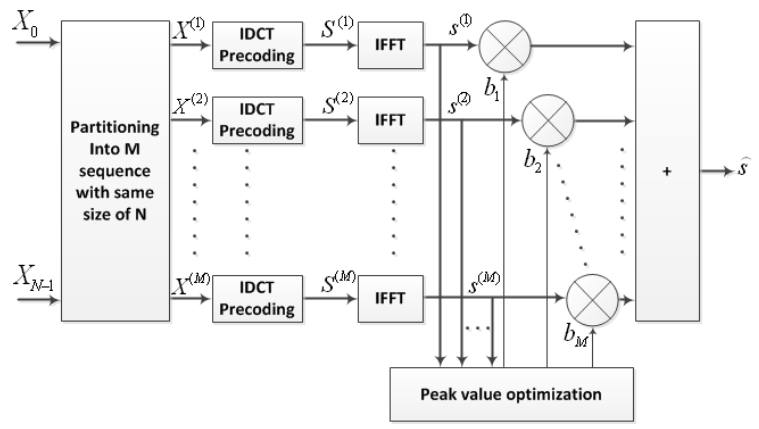

Fig. 2 The block diagram of the proposed method.

basically try all possible combinations of rotation factors to find the vector of the best rotation factors $b=\left[b_{1}, b_{2}, \ldots, b_{M}\right]$. With the PTS approach it is recommended to leave the first partial output unrotated (i.e., $b_{1}=1$ ) for more freedom and hence the maximum number of iterations to find the best values for the $b$ vector is $W^{M-1}$.

The performance of the PTS approach is proportional to $M$ and $W$ which means that the complexity should be increased for better PAPR reduction results.

\section{PROPOSED METHOD}

In this method, we propose using the IDCT as precoder for the PTS subblocks to spread each value of the partition data sequence over all subcarriers before applying the IFFT function. The proposed technique is illustrated in the block diagram shown in Fig. 2. The only difference between this approach and the PTS approach is the addition of the IDCT precoder blocks. The coded sequence $S^{(m)}$ is given by

$$
S^{(m)}=\operatorname{IDCT}\left(X^{(m)}\right)=C^{-1} X
$$

where $C^{-1}$ is the $N \times N$ matrix of the IDCT:

$$
\mathrm{C}_{i j}^{-1}= \begin{cases}1 / \sqrt{N}, & j=0, \\ \sqrt{2 / N} \cos ((2 i+1) j \pi / 2 N), & \text { otherwise, }\end{cases}
$$

where $i, j=0,1, \ldots, N-1$ and represent the rows and columns indices, respectively.

\section{RESULTS AND DISCUSSION}

The proposed method was tested for $N=64,128$ and 256 and 4-QAM for symbol mapping. The phase factor range used in simulation test was $\{ \pm 1, \pm j\}$ for $M=2$ and 4 and $\{ \pm 1\}$ for $M=4$ and 8 . In other words, $(2,4),(4,2),(4,4)$ and $(8,2)$ are the four combinations of $(M, W)$ that were tested in the simulation. The CCDF was calculated for $10^{4}$ frames 


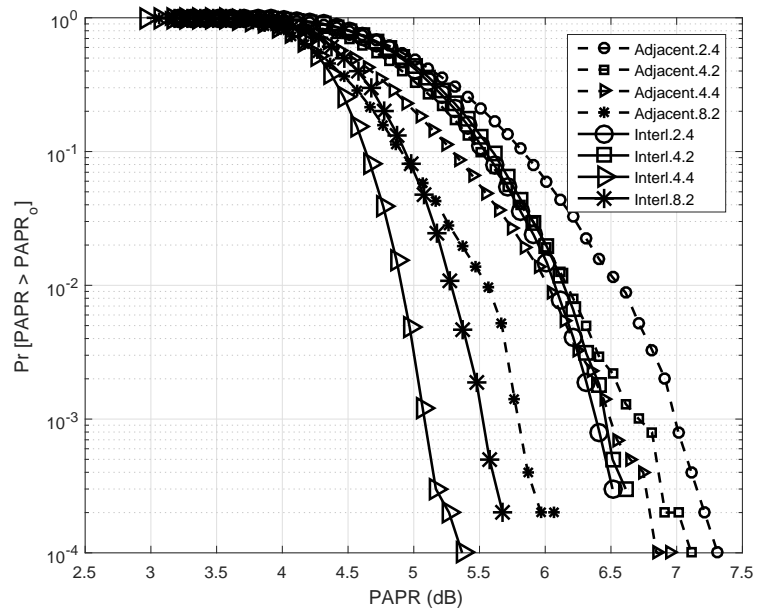

Fig. 3 CCDF plot for comparison between interleaved and adjacent partitioning scheme of proposed method with 4-QAM and 64 subcarriers.

of data and the PAPR is calculated for the OFDM output signal generated using $L=1$.

Before going ahead with the comparison between the PTS and the proposed methods, a test has been made to decide which type of partitioning is better for the proposed approach. It is already known from Ref. 17 that the adjacent partitioning is better than interleaved partitioning for the PTS method. However, the results in Fig. 3 show that the proposed technique herein has a better PAPR reduction performance with interleaved partitioning than with adjacent partitioning. Hence the comparison has been conducted between the PTS and proposed methods with adjacent and interleaved partitioning, respectively.

It can be seen clearly from the CCDF plot in Fig. 4 that the proposed method has better PAPR reduction results than with the PTS method. Also, as seen in Table 1, the proposed method was able to reduce the OFDM PAPR 2.6 times more than the PTS PAPR reduction result for $N=64$, 4-QAM, $M=2$ and $W=4$ with $W^{M-1}=4$ iterations only.

It is clear from the results that the performance of the proposed technique is proportional to the number of partitions and admitted angles. However, the impact of one parameter could be greater than another such as for $(8,2)$ and $(4,4)$ where the impact of increasing the number of admitted angles is greater than the impact of decreasing the number of partitions.

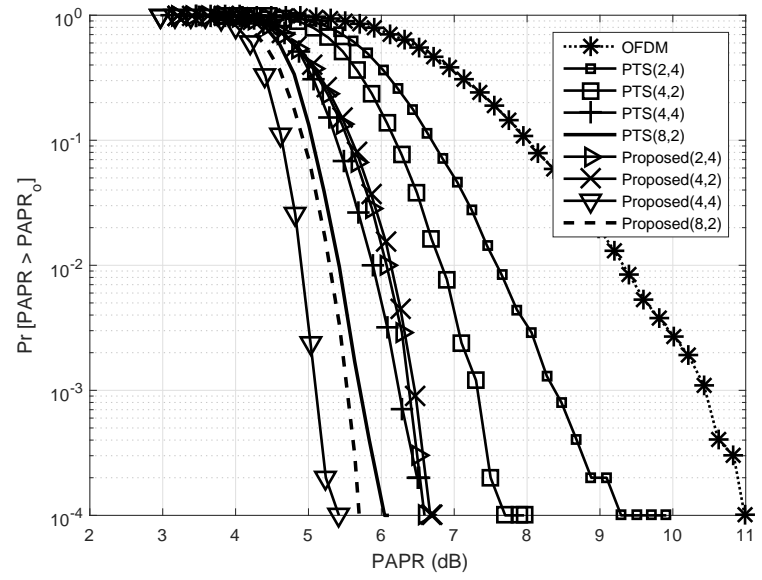

Fig. 4 CCDF plot for comparison between the proposed method, the conventional PTS method, and OFDM with 4-QAM, 64 subcarriers, and various $M$ and $W$ values.

Table 1 CCDF values at a clip rate of $10^{-4}$ for 4-QAM.

\begin{tabular}{|c|c|c|c|c|c|}
\hline \multirow[b]{2}{*}{$N$} & \multirow[b]{2}{*}{ M.W } & \multirow{2}{*}{$\begin{array}{l}\text { OFDM } \\
(\mathrm{dB})\end{array}$} & \multicolumn{2}{|c|}{$\begin{array}{l}\text { PAPR Reduction } \\
\text { (dB) }\end{array}$} & \multirow{2}{*}{$\begin{array}{c}\begin{array}{c}\text { Improvement } \\
\text { over PTS (\%) }\end{array} \\
\text { Proposed }\end{array}$} \\
\hline & & & PTS & Proposed & \\
\hline \multirow[t]{4}{*}{64} & 2.4 & 10.98 & 1.709 & 4.398 & 257.3 \\
\hline & 4.2 & & 3.38 & 4.318 & 127.8 \\
\hline & 4.4 & & 4.469 & 5.682 & 127.1 \\
\hline & 2.4 & & 5.076 & 5.284 & 104.1 \\
\hline \multirow[t]{4}{*}{128} & 2.4 & 11.22 & 1.776 & 4.298 & 242 \\
\hline & 4.2 & & 2.688 & 4.306 & 160.2 \\
\hline & 4.4 & & 3.975 & 5.561 & 139.9 \\
\hline & 2.4 & & 4.62 & 5.306 & 114.9 \\
\hline \multirow[t]{4}{*}{256} & 2.4 & 11.83 & 2.234 & 4.663 & 208.7 \\
\hline & 4.2 & & 2.956 & 4.647 & 157.2 \\
\hline & 4.4 & & 4.156 & 5.713 & 137.5 \\
\hline & 2.4 & & 4.596 & 5.573 & 121.3 \\
\hline
\end{tabular}

\section{CONCLUSIONS}

An IDCT-based precoder with PTS technique was proposed in this paper. The proposed method was tested with various numbers of subcarriers, various numbers of partitions and two group of admitted angles. The proposed method showed an improvement in terms of PAPR reduction over all configured parameters of $N, M$, and $W$. The simulation results were consistent and directly proportional to the number of partitions and admitted angles. We conclude that the proposed technique surpassed the PTS method and the use of the IDCT algorithm improved the performance of the PTS concept in terms of PAPR reduction. 
Acknowledgements: The authors would like to thank the referees and editors for providing very helpful comments and suggestions. This project was supported by Research University Grant, Universiti Sains Malaysia (1001/PELECT/814245).

\section{REFERENCES}

1. Müller SH, Bäuml RW, Fischer RF, Huber JB (1997) OFDM with reduced peak-to-average power ratio by multiple signal representation. Ann Telecomm 52, 58-67.

2. Müller SH, Huber JB (1997) A novel peak power reduction scheme for OFDM. In: The 8th IEEE International Symposium on Personal, Indoor and Mobile Radio Communications, vol 3, pp 1090-4.

3. Muller S, Huber J (1997) OFDM with reduced peakto-average power ratio by optimum combination of partial transmit sequences. Electron Lett 33, 368-9.

4. Jayalath ADS, Tellambura C (2000) The use of interleaving to reduce the peak-to-average power ratio of an OFDM signal. In: IEEE Global Telecommunications Conference, vol 1, pp 82-6.

5. Hwang CS (2001) A peak power reduction method for multicarrier transmission. In: IEEE International Conference on Communications, vol 5, pp 1496-500.

6. Wattanasuwakull T, Benjapolakul W (2005) PAPR Reduction for OFDM transmission by using a method of tone reservation and tone injection. In: The 5th International Conference on Information, Communications and Signal Processing, pp 273-7.

7. Park SE, Yun S, Kim JY, Park DS, Joo P (2003) Tone reservation method for PAPR reduction scheme. In: Project IEEE 802.16 Working Group on Broadband Wireless Access Standards, Contributed Document IEEE C802.16e-03/60.

8. Tellado J, Cioffi JM (1998) Peak power reduction for multicarrier transmission. In: IEEE Globecom, vol 99, pp 5-9.

9. Yang G, Yang Z, Xu J (2007) PAPR reduction in the OFDM system employing Tone Reservation based on FFT/IFFT. In: International Symposium on Communications and Information Technologies, pp 91-4.

10. Zhou J, Qiao Y (2015) Low-PAPR asymmetrically clipped optical OFDM for intensity-modulation/ direct-detection systems. IEEE Photonics $J$ 7, 1-8.

11. Pennebaker WB, Mitchell JL (1993) JPEG: Still Image Data Compression Standard, Springer Science \& Business Media.

12. Baig I, Jeoti V (2010) DCT precoded SLM technique for PAPR reduction in OFDM systems. In: International Conference on Intelligent and Advanced Systems, pp 1-6.

13. Wahab A, Sa'd AHY (2017) An improved IDCT-OFDM with partial transmit sequence. In: 9th International Conference on Robotic, Vision, Signal Processing and Power Applications, pp 535-46.
14. Wang Z (2010) Reduction PAPR of OFDM signals by combining SLM with DCT transform. Int $J$ Comm Netw Syst Sci 3, 888-92.

15. Wang Z, Chen S, et al (2014) Reduction PAPR of OFDM signals by combining grouped DCT precoding with PTS. J Signal Inform Process 5, 135-42.

16. Zhou J, Xu F, Sun E, Zhang T, Zhang Z, Guo M, Tang X, Wang Z, et al (2016) Coherent optical interleaved SC-FDM uplink scheme for long-reach passive optical network. IEEE Photonics $J$ 8, 1-8.

17. Varahram P, Ali BM (2011) Partial transmit sequence scheme with new phase sequence for PAPR reduction in OFDM systems. IEEE Trans Consum Electron 57, 366-71. 